\title{
Pathophysiology and preventive strategies of anthracycline-induced cardiotoxicity
}

\author{
Woo-Baek Chung and Ho-Joong Youn
}

Division of Cardiology, Department of Internal Medicine, College of Medicine, The Catholic University of Korea, Seoul, Korea

Received: January 17, 2016

Accepted: May 20, 2016

\section{Correspondence to}

Ho-Joong Youn, M.D.

Cardiovascular Center, Division of Cardiology, Department of Internal Medicine, College of Medicine, Seoul St. Mary's Hospital, The Catholic University of Korea, 222 Banpo-daero, Seocho-gu, Seoul o6591, Korea Tel: +82-2-2258-6029 Fax: +82-2-591-1506 E-mail: younhj@catholic.ac.kr
Cardiotoxicity is a well-known complication following treatment with anthracyclines. However, they are still widely used in chemotherapy for breast cancer, lymphoma, leukemia, and sarcoma, among others. Patient clinical characteristics, such as age, sex, comorbidities, anthracycline dose and infusion schedule, and the combined anti-cancer agents used, are diverse among cancer types. It is difficult to recommend guidelines for the prevention or management of anthracycline-induced cardiotoxicity applicable to all cancer types. Therefore, anthracycline-induced cardiotoxicity remains a major limitation in the proper management of cancer patients treated with an anthracycline-combined regimen. Efforts have been extensive to determine the mechanism and treatment of anthracycline-induced cardiotoxicity. Because cardiotoxicity causes irreversible damage to the myocardium, prevention is a more effective approach than treatment of cardiotoxicity after symptomatic or asymptomatic cardiac dysfunction develops. This article will review the pathophysiological mechanisms of anthracycline-induced cardiotoxicity and strategies for protecting the myocardium from anthracycline.

Keywords: Cardiotoxicity; Anthracyclines; Doxorubicin; Drug therapy; Neoplasms

\section{INTRODUCTION}

The 5-year survival rate of cancer patients in Korea has increased by $68.1 \%$; consequently, the estimated number of patients who underwent cancer treatment was up to 1.37 million people in 2013 [1]. The number of patients receiving chemotherapy and surviving after chemotherapy is also gradually increasing. Caution is required for most anti-cancer agents currently in use due to various adverse effects, and cardiotoxicity is a well-known major adverse effect that impacts the quality of life and mortality of cancer patients [2]. The development of cardiotoxicity during chemotherapy limits the proper treatment of cancer itself and thus deteriorates the quality of life if it results in overt heart failure.

Cardiotoxicity can increase mortality not only due to cancer itself but also due to heart failure even if the patient has survived cancer. Many types of cardiotoxicity have been reported, including bradycardia, QT interval prolongation, myocardial ischemia, and cardiomyopathy [3], and one type of anti-cancer drug may cause various types of cardiotoxicity [2-4]. The pathogenesis of cardiotoxicity is different for each drug. Additionally, the pathogenesis of anthracycline-induced cardiotoxicity has not yet been clearly determined; this lack of knowledge is a major obstacle to the prevention and treatment of cardiotoxicity.

Although the anthracyclines are representative chemotherapeutic agents that can cause cardiotoxicity, particularly left ventricular dysfunction, they are very effective chemotherapeutic agents for use in treating breast cancer, lymphoma, leukemia, and sarcoma, among others. 
The early generation anthracycline compounds daunorubicin and doxorubicin can cause fatal heart failure [5]. Anthracyclines developed later, such as idarubicin, epirubicin, and mitoxantron, are less cardiotoxic than the earlier generation drugs, but cardiotoxicity is still a problem. Doxorubicin, an anthracycline compound, has been studied extensively, and the incidence of cardiotoxicity increases according to the cumulative dose [6]. The development of subclinical cardiotoxicity at a cumulative doxorubicin dose less than $300 \mathrm{mg} / \mathrm{m}^{2}$ was recently reported [7].

There has been tremendous effort to understand the pathophysiology of anthracycline-induced cardiotoxicity and research to find an appropriate treatment, but no effective preventive drug has been developed for use in clinical practice. Because anthracycline-induced cardiotoxicity can cause irreversible damage to the myocardium [6], the early detection of cardiotoxicity and prevention of progression to heart failure is the most effective strategy [7-9]. In clinical practice, each cancer type has different clinical characteristics, dosing schedules, and combination regimens; therefore, it is difficult to suggest a recommendation that can be applied to any cancer type. The current understanding of the pathophysiology of anthracycline-induced cardiotoxicity and awareness of monitoring, prevention, and treatment strategies are important in the management of patients receiving anthracyclines.

\section{DEFINITION OF CARDIOTOXICITY}

The most commonly accepted definition of cardiotoxicity in the oncology community is an absolute decrease of $10 \%$ or more in the left ventricular ejection fraction (LVEF) from baseline or an LVEF decline to < $50 \%$ compared with the baseline [10]. However, some studies were conducted with a normal cut-off value of $55 \%$ of LVEF, as measured by echocardiography $[11,12]$. Cardiotoxicity is also defined as a LVEF decline of $\geq 5 \%$ to $<55 \%$ with heart failure symptoms or an asymptomatic decrease in LVEF of $\geq 10 \%$ to $<55 \%$ in patients treated with trastuzumab, a monoclonal antibody for the treatment of breast cancer [13]. Therefore, physicians may be confused as there is not yet a consensus definition for cardiotoxicity that can be applied to all cancer types.
There are also imaging modality discrepancies in the measurement of LVEF. Multigated radionuclide angiography (MUGA) is reliable and was commonly used for the evaluation of LVEF in anthracycline-induced cardiotoxicity before two-dimensional (2D) echocardiography became the gold-standard method for measuring LVEF. A comparative study demonstrated that the average LVEF measured by MUGA was less than that of echocardiography in the same group of patients [14], suggesting that the choice of imaging modality can affect the detection of cardiotoxicity. It is reasonable to define cardiotoxicity by the consensus of the oncologist and cardiologist. Clearly, the baseline measurement of LVEF before chemotherapy is most important in the evaluation of anthracycline-induced cardiotoxicity.

\section{PATHOPHYSIOLOGY OF ANTHRACYCLINE-IN- DUCED CARDIOTOXICITY}

The histological pathophysiology of anthracycline-induced cardiotoxicity is characterized by myocardial damage due to proteolysis, necrosis, apoptosis, and fibrosis. Proteolysis is a relatively acute response to anthracycline treatment. Treatment of cultured adult rat cardiomyocytes with doxorubicin for 24 hours resulted in the degradation of titin, the largest myofilament protein [15]. This is an early event after doxorubicin treatment, and proteolysis of the spring-like domain of titin may predispose cardiomyocytes to diastolic dysfunction and myofilament instability [15]. Electron microscopic examination of a doxorubicin-induced cardiotoxicity rat model
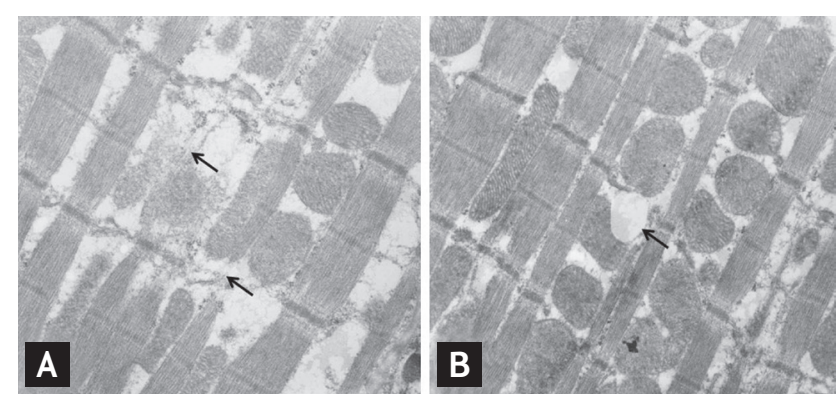

Figure 1. Electron microscopic images of the left ventricle of doxorubicin-treated rats $(\times 13,200)$. (A) Disrupted myofibrils (upper arrow) and loss of Z-bands (lower arrow). (B) Vacuolation of mitochondria (arrow). Modified from Choi et al. [16]. 


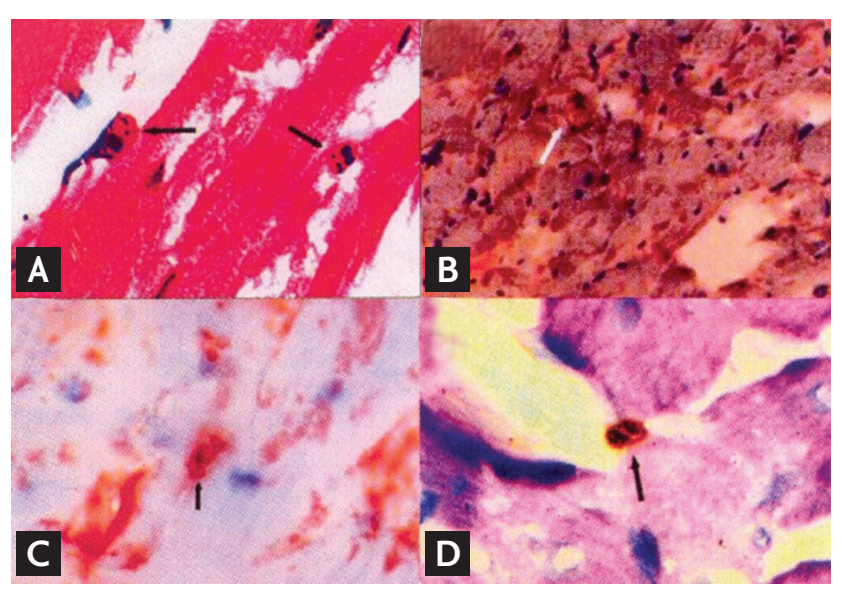

Figure 2. Histological changes of the myocardium after doxorubicin treatment (×400). (A) H\&E. (B) Immunohistochemical staining for Bax. (C) Immunohistochemical staining for caspase 3. (D) TUNEL (terminal deoxynucleotidyl transferase dUTP nick end labelin) assay. Arrows of each stains demonstrates fragmented nuclei of apoptotic cells. Adapted from Choi et al. [17].

demonstrated the destruction of the microstructures of myocardial cells, including myofilament dropout (Fig. 1A, upper arrow), loss of the Z-band (Fig. 1A, lower arrow), and vacuolation of mitochondria (Fig. $1 B$, arrow) [16]. These changes result in the dysfunction of myocardial cells and even cell death via necrosis.

Apoptosis is another important mechanism involved in myocardial damage. After intraperitoneal injection of doxorubicin for 2 weeks, light microscopic examination of the rat myocardium revealed nuclear fragmentation and chromatin condensation by H\&E staining (Fig. 2A) and Bax- (Fig. 2B), caspase 3- (Fig. 2C), and TUNEL (terminal deoxynucleotidyl transferase dUTP nick end labelin) assay- (Fig. 2D) positive apoptotic cells [17]. After the intraperitoneal injection of doxorubicin for 6 weeks in a chronic exposure animal model, the fibrosis burden was increased in the interstitial space of the myocardium of doxorubicin-treated normotensive Wistar-Kyoto rats (Fig. ${ }_{3}$ C) compared with control rats (Fig. 3A). The most extensive fibrotic burden was observed in the interstitial space of doxorubicin-treated spontaneously hypertensive rats (Fig. 3D) compared with the controls (Fig. $3 \mathrm{~B}$ ), suggesting that hypertension is a definite risk factor for doxorubicin-induced myocardial damage [18]. The same pattern of changes was also observed in the perivascular area. Early cell death and the delayed increment of myocardial fibrosis are major histological

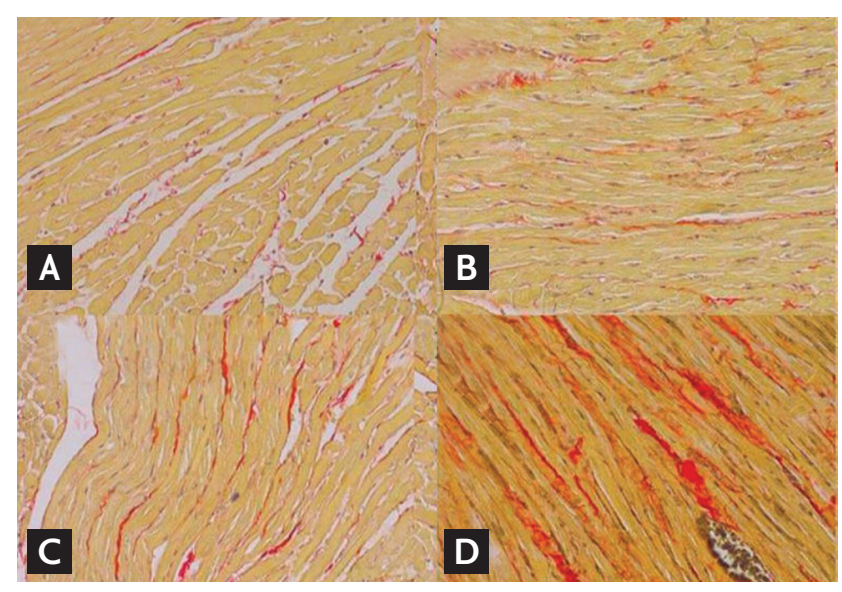

Figure 3. Picrosirius red staining for collagen fibers in the rat myocardium (×400). (A) Interstitial area of Wistar-Kyoto rats (WKYs). (B) Interstitial area of spontaneously hypertensive rats (SHRs). (C) Interstitial area of doxorubicin-treated WKYs. (D) Interstitial area of doxorubicin-treated SHRs. Modified from Uhm et al. [18].

changes that eventually result in both systolic and diastolic cardiac dysfunction.

The molecular mechanism of cardiotoxicity has also been studied extensively. The most widely accepted mechanism is the generation of reactive oxygen species (ROS), related to oxidative stress [19]. During the metabolism of anthracyclines, unpaired electrons can be rapidly transferred to an oxygen molecule resulting in ROS generation [20]. The generation of superoxide anions by anthracycline metabolism can cause subsequent cellular damage by the degradation of the sarcomere, mitochondrial dysfunction, and DNA damage [21,22]. The reduction of the carbonyl group of anthracyclines generates toxic metabolites at the myocardium level [23]. The accumulation of toxic metabolites inhibits the calcium and sodium exchange pumps in the mitochondrial membrane, induces disturbance in the myocardial energetics and eventually systolic dysfunction [24]. Anthracyclines can also alter the iron homeostasis through the creation of Fe-anthracycline complexes, subsequently producing ROS [25]. Oxidative stress also mediates mitochondrial dysfunction by cardiolipin, a phospholipid with an important role in energy metabolism and a component of the inner membrane of mitochondria [26].

Anthracyclines can facilitate the release of pro-inflammatory cytokines by stimulating macrophages [27], which play a role in the development of cardiotoxici- 


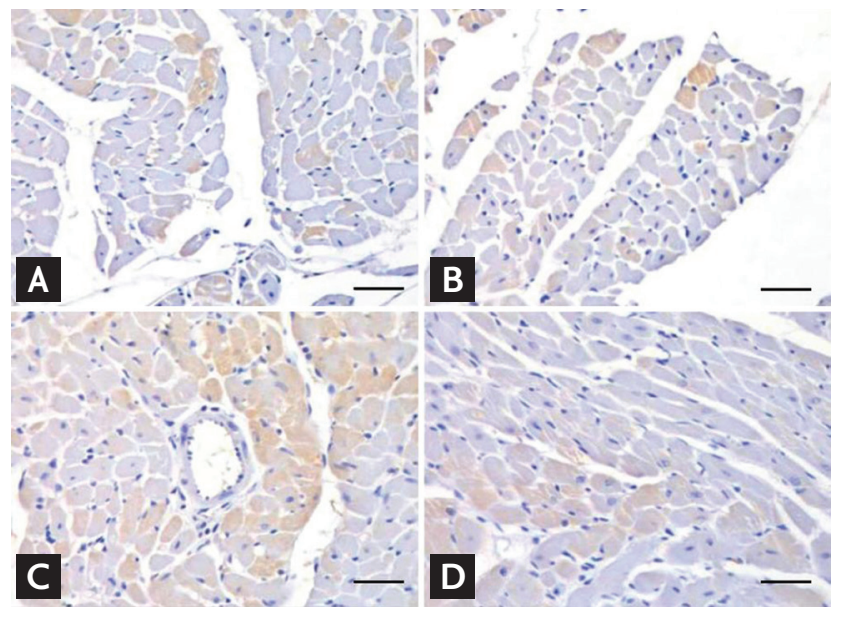

Figure 4. Immunohistochemical staining of the myocardium. The scale bars indicate $25 \mu \mathrm{m}$. The brown-colored cells are the cardiac ankyrin repeat protein (CARP)-expressing cells that were detected by the CARP antibody. (A) Normal saline-treated Wistar-Kyoto rat (WKY) myocardium. (B) Adriamycin-treated WKY myocardium. (C) Normal saline-treated spontaneously hypertensive rat (SHR) myocardium. (D) Adriamycin-treated SHR myocardium. Adapted from Chung et al. [32].

ty. Interleukin (IL)-1 $\beta$ and IL-6 expression is increased in doxorubicin-treated cells, without changes in the expression of tumor necrosis factor (TNF). These cytokines mainly modulate apoptosis through TNF receptors, whose function is affected by doxorubicin [28]. According to our previous study, p53 could promote apoptosis by a caspase-independent pathway, and doxorubicin induced $\mathrm{p}_{53}$ expression in $\mathrm{H}_{9} \mathrm{c} 2$ cardiomyocytes [29]. Topoisomerase (Top) $2 \beta$, a recently suggested primary mediator for anthracycline-induced cardiotoxicity, is required for $\$ 53$ activation in response to anthracycline-induced DNA damage in cardiomyocytes [30]. Top $2 \beta$ also reduced antioxidant enzyme gene transcription and induced ROS production [30] and deletion of the Top $2 \beta$ gene protected the myocardium from anthracycline-induced cardiotoxicity in a mouse model [31].

Anthracycline-sensitive cardiac ankyrin repeat protein (CARP), a transcriptional regulatory protein that negatively regulates the expression of cardiac genes, is another molecule with a role in anthracycline-induced cardiotoxicity [32]. The expression of CARP in cardiac myocytes of spontaneously hypertensive rats was also reported (Fig. 4), suggesting that hypertension upregulates CARP expression. However, the role of CARP in the anthracycline-induced cardiotoxicity of hypertensive patients is unclear.

Damage to intracellular molecules by ROS and toxic metabolites of anthracyclines, the modulation of pro-inflammatory cytokines, and the interaction of anthracyclines with intracellular proteins such as Top $2 \beta$ and CARP can lead to cardiomyocyte death. Because cell death plays an important role in the development of anthracycline-induced cardiotoxicity, the myocardial cell number determined during the embryonic development period can be an independent factor for the susceptibility of cardiac dysfunction [33]. Survivin, a key regulator of mitotic progression, plays a crucial role in controlling the cardiomyocyte number during embryonic development and adult life through its profound impact on cardiomyocyte replication and may thus emerge as a new target for myocardial regeneration [33].

\section{STRATEGIES FOR CARDIAC FUNCTION MONI- TORING}

The cumulative dose of anthracycline is the most important risk factor for the development of cardiotoxicity. The estimated heart failure incidences were $5 \%, 16 \%$, and $26 \%$ for cumulative doses of 400,500 , and $550 \mathrm{mg} /$ $\mathrm{m}^{2}$ doxorubicin, respectively [34], and the limitation of the cumulative anthracycline dose is 400 to $450 \mathrm{mg} / \mathrm{m}^{2}$. The risk of cardiac events after chemotherapy is higher than tumor recurrence, with a 10-fold higher rate of cardiovascular disease and 15-fold increased rate of heart failure than in the general population [35]. Subclinical cardiotoxicity can develop during chemotherapy (Fig. 5) [7,36]; some patients may recover from it, while others may progress to overt heart failure [36].

Although it is not clear which clinical factor differs between the recovering group and progress-to-heart failure group, it is important to detect subclinical cardiotoxicity during the chemotherapeutic period. The cumulative incidences of cardiac events peaked at 1 year after the completion of anthracycline treatment [37,38]. There are insufficient data to determine the incidence of late-onset cardiotoxicity [2], which is defined as the development of cardiac events more than 1 year after the completion of anthracycline treatment. A significantly higher incidence of cardiovascular risk factors, such as 


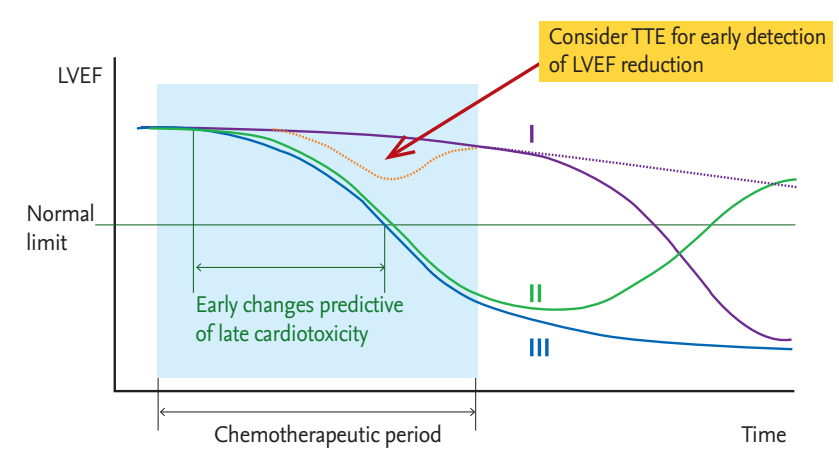

Figure 5. Schematic representation of changes in the left ventricular ejection fraction (LVEF) and timing of the detection of subclinical cardiotoxicity. (I) Late onset cardiotoxicity (radiotherapy, anthracyclines), (II) reversible cardiotoxicity (trastuzumab), and (III) irreversible cardiotoxicity during treatment (anthracyclines). TTE, transthoracic echocardiogram. Modified from Altena et al. [36], with permission from Elsevier.

hypertension, diabetes, dyslipidemia, and obesity in survivors of childhood cancer has been reported, and these factors may play a role in the development of late-onset cardiotoxicity [39]. Patients who already have cardiovascular risks before chemotherapy require more attention for cardiotoxicity in the course of treatment.

Because anthracycline-induced cardiotoxicity is suggested to cause irreversible damage to the myocardium (Fig. 5, line III) [36,40], the proper monitoring of cardiac function and early detection of cardiotoxicity are crucial. The assessment of baseline cardiac function before starting chemotherapy is the first step in monitoring. By definition, the detection of reduced LVEF compared with the baseline is a critical step in the diagnosis of cardiotoxicity. MUGA and echocardiography are the most studied methods for the measurement of LVEF. MUGA is somewhat insensitive for the detection of subtle changes in cardiac function [36]. Therefore, echocardiography is now the gold standard for screening and early detection of subclinical cardiotoxicity. Echocardiography has advantages in the assessment of diastolic dysfunction that can develop before systolic dysfunction; tissue velocity imaging and deformation imaging techniques, such as the strain and strain rate in both $2 \mathrm{D}$ and $3 \mathrm{D}$ echocardiography, can detect early subclinical changes in myocardial function [41,42].

Recently, cardiac magnetic resonance imaging (MRI) has been increasingly used for the evaluation of cardiotoxicity due to its accuracy and reproducibility in the measurement of the ventricular volume and LVEF [43]. MRI can also provide an opportunity for the detection of myocardial inflammation and edema during the early stages of cardiac injury and the presence of myocardial fibrosis in the late stages [44]. The limitation of using MRI for cardiac function monitoring is that it may not be cost effective for repeated assessments.

Circulating biomarkers, such as troponin and braintype natriuretic peptide (BNP) are widely studied for use in the monitoring of cardiotoxicity. Troponin, the most widely studied biomarker for the detection of cardiotoxicity; it is recommended that troponin levels are evaluated at baseline and during and after chemotherapy [13]. The early elevation of troponin (i.e., within 72 hours after each cycle) and sustained elevation (i.e., 1 month after treatment) suggest the highest cardiotoxicity event rate [45]. However, the role of BNP in the prediction and diagnosis of cardiotoxicity remains unclear [46].

The 2012 European Society of Medical Oncology (ESMO) Clinical Practice Guidelines for cardiovascular toxicity [13] recommended management algorithms for anthracycline-induced cardiotoxicity. For cardiac function monitoring, it was recommended that the first follow-up echocardiogrphy should be performed the end of chemotherapy and not at the cut-off cumulative dose of anthracycline. However, as the development of subclinical cardiotoxicity can occur at less than $300 \mathrm{mg} /$ $\mathrm{m}^{2}$ of the cumulative dose of doxorubicin [7], cardiac function monitoring should be performed before the completion of anthracycline treatment. Although it was experience of only three hospitals in Korea, this study [7] demonstrated that, at the cumulative dose of doxorubicin of $244.5 \mathrm{mg} / \mathrm{m}^{2}$, the subclinical cardiotoxicity could be predicted (sensitivity, 71.4\%; specificity, 70.9\%; area under the curve, $0.741 ; 95 \%$ confidence interval, 0.608 to $0.874 ; p=0.001$ ) and suggested that performing early monitoring before reaching the $300 \mathrm{mg} / \mathrm{m}^{2}$ cumulative dose of doxorubicin might be a proper strategy for the early detection of anthracycline-induced cardiotoxicity. Additionally, in the same study, the enforcement rate of cardiac function monitoring with echocardiography was very low previously, only about 5 to 6 years ago; therefore, physicians should pay attention to cardiac function monitoring during the chemotherapeutic period. The guidelines also recommend an annual follow-up with echocardiography 1 year after the completion of anth- 


\section{KJIM}

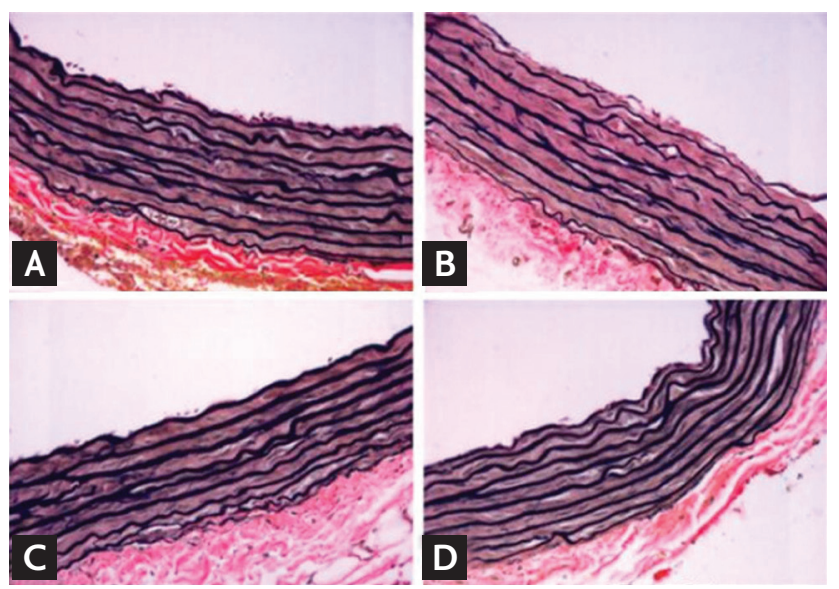

Figure 6. Verhoff's elastin-stained section of the aorta of Wistar-Kyoto rats (×400). (A) Control group, (B) adriamycin-treated (AD) group, (C) candesartan-treated (CA) group, and (D) AD+CA group. Modified from Uhm et al. [49].

racycline treatment but did not recommend how long cardiac function should be followed-up after chemotherapy.

A recent cardiac function monitoring study employed MRI and echocardiography 18 years after the completion of anthracycline treatment and demonstrated that the incidence of $\mathrm{LVEF}<50 \%$ was $16 \%$ with both methods [47]. Considering that the patients received a relatively high dose of epirubicin, the incidence of cardiotoxicity was lower than early-onset cardiotoxicity within 1 year after completion of chemotherapy. This result indicates that some patients still need long-term monitoring of cardiac function, and further studies are mandatory to determine which patients and how long cardiac function should be monitored.

\section{STRATEGIES FOR THE PROTECTION OF CAR- DIAC FUNCTION}

There are strategies for the protection of cardiac function during anthracycline treatment: primary and secondary prevention [4]. Primary strategies for the prevention of cardiotoxicity include the continuous infusion of anthracycline and the use of liposomal doxorubicin and the cardioprotective agent dexrazoxane. The continuous infusion of doxorubicin for 48 to 72 hours is effective for cardioprotection in sarcoma and lymphoma but not in pediatric patients. Liposomal doxorubicin is approved for limited cancer types. Dexrazoxane is approved only for women with metastatic breast cancer who received at least $300 \mathrm{mg} / \mathrm{m}^{2}$ doxorubicin and need additional doxorubicin for the maintenance of tumor control [4]. In animal studies, the concomitant administration of insulin-like growth factor 1 reduced apoptosis in doxorubicin-treated mouse myocardium [48], and candesartan prevented elastin degradation (Fig. 6) in doxorubicin-treated rat aorta [49]. In humans, many agents were studied for a possible cardioprotective effect against anthracycline-induced cardiotoxicity (Table 1) [50]; however, there are still limited data [48]. Secondary prevention strategies including the early detection and treatment of left ventricular dysfunction are suggested by the 2012 ESMO guidelines [13]. These guidelines recommended the aggressive treatment of left ventricular dysfunction even in asymptomatic patients after anthracycline therapy, particularly when the patient has a good chance for long-term survival.

Angiotensin-converting enzyme inhibitors, angiotensin receptor blockers, and $\beta$-blockers should be administered, and the earlier heart failure therapy is initiated (within 2 months from the end of anthracycline therapy), the better the therapeutic response will be [13]. Although starting treatment is recommended if left ventricular dysfunction is detected, many cancer survivors are not receiving treatment consistent with heart failure guidelines [11]. Oncologists should pay more attention to cardiac monitoring, and cardiologists should focus more on the proper treatment of anthracycline-induced cardiotoxicity.

\section{CONCLUSIONS}

Understanding the pathophysiology of anthracycline-induced cardiotoxicity is important to determine the therapeutic target of cardiotoxicity and for the development of protective agents. Drugs used in current clinical practice, such as dexrazoxane, $\beta$-blockers, angiotensin-converting enzyme inhibitors, and angiotensin receptor blockers, do not exert sufficient cardioprotective effects in anthracycline-induced cardiotoxicity; however, there are ongoing studies to evaluate proper treatment strategies. Cardiac function monitoring in the chemotherapeutic period is an important step for the early detection of anthracycline-induced cardiotoxicity, and 
Table 1. Evaluated cardioprotective agents in humans

\begin{tabular}{lll}
\hline Agent & \multicolumn{1}{c}{ Class } & \multicolumn{1}{c}{ Mechanism } \\
\hline Carvedilol & $\beta$-Adrenergic antagonist & $\begin{array}{l}\text { Prevention of free radical formation; prevention of depletion of } \\
\text { endogenous anti-oxidants }\end{array}$ \\
Valsartan & Angiotensin II receptor blocker & $\begin{array}{l}\text { Inhibition of angiotensin II effects } \\
\text { Increase of nitric oxide release }\end{array}$ \\
Dexrazoxane & Chelating agent & $\begin{array}{l}\text { Prevention of free radical formation; binding to iron inhibits } \\
\text { DNA topoisomerase }\end{array}$ \\
Co-enzyme Q10 & Dietary supplement & Anti-oxidant \\
Carnitine & Dietary supplement & Anti-oxidant; transfer of long chain fatty acids into mitochondria \\
N-acetylcysteine & Mucolytic agent & Promotion of endogenous antioxidant synthesis \\
Vitamins A, C, E & Nutrient & Anti-oxidant \\
\hline
\end{tabular}

Adapted from Cardinale et al. [50], with permission from Elsevier.

treatment at the proper time can prevent progression to overt heart failure after anthracycline therapy to increase the odds of long-term survival. Collaboration between oncologists and cardiologists is necessary to improve the management of cancer patients receiving anthracyclines.

\section{Conflict of interest}

No potential conflict of interest relevant to this article was reported.

\section{REFERENCES}

1. Jung KW, Won YJ, Oh CM, et al. Prediction of cancer incidence and mortality in Korea, 2015. Cancer Res Treat 2015;47:142-148.

2. Yeh ET, Bickford CL. Cardiovascular complications of cancer therapy: incidence, pathogenesis, diagnosis, and management. J Am Coll Cardiol 2009;53:2231-2247.

3. Albini A, Pennesi G, Donatelli F, Cammarota R, De Flora S, Noonan DM. Cardiotoxicity of anticancer drugs: the need for cardio-oncology and cardio-oncological prevention. J Natl Cancer Inst 2010;102:14-25.

4. Kim SM, Kwak CH, Lee B, et al. A case of severe coronary spasm associated with 5-fluorouracil chemotherapy. Korean J Intern Med 2012;27:342-345.

5. Vejpongsa P, Yeh ET. Prevention of anthracycline-induced cardiotoxicity: challenges and opportunities. J Am Coll Cardiol 2014;64:938-945.

6. Angsutararux P, Luanpitpong S, Issaragrisil S. Chemo- therapy-induced cardiotoxicity: overview of the roles of oxidative stress. Oxid Med Cell Longev 2015;2015:795602.

7. Chung WB, Yi JE, Jin JY, et al. Early cardiac function monitoring for detection of subclinical doxorubicin cardiotoxicity in young adult patients with breast cancer. J Breast Cancer 2013;16:178-183.

8. Sawaya H, Sebag IA, Plana JC, et al. Early detection and prediction of cardiotoxicity in chemotherapy-treated patients. Am J Cardiol 2011;107:1375-1380.

9. Cardinale D, Colombo A, Bacchiani G, et al. Early detection of anthracycline cardiotoxicity and improvement with heart failure therapy. Circulation 2015;131:1981-1988.

10. National Cancer Institute. Common Terminology Criteria for Adverse Events (CTCAE) version 4.0 [Internet]. Bethesda (MD): National Cancer Institute, c2009 [cited 2016 May 14]. Available from: http://ctep.cancer.gov/protocolDevelopment/ electronic_applications/ctc.htm.

11. Yoon GJ, Telli ML, Kao DP, Matsuda KY, Carlson RW, Witteles RM. Left ventricular dysfunction in patients receiving cardiotoxic cancer therapies are clinicians responding optimally? J Am Coll Cardiol 2010;56:1644-1650.

12. Toro-Salazar OH, Ferranti J, Lorenzoni R, et al. Feasibility of echocardiographic techniques to detect subclinical cancer therapeutics-related cardiac dysfunction among high-dose patients when compared with cardiac magnetic resonance imaging. J Am Soc Echocardiogr 2016;29:119131.

13. Curigliano G, Cardinale D, Suter T, et al. Cardiovascular toxicity induced by chemotherapy, targeted agents and radiotherapy: ESMO Clinical Practice Guidelines. Ann Oncol 2012;23 Suppl 7:vii155-viiı66. 
14. Corapcioglu F, Sarper N, Berk F, Sahin T, Zengin E, Demir H. Evaluation of anthracycline-induced early left ventricular dysfunction in children with cancer: a comparative study with echocardiography and multigated radionuclide angiography. Pediatr Hematol Oncol 2006;23:71-80.

15. Lim CC, Zuppinger C, Guo X, et al. Anthracyclines induce calpain-dependent titin proteolysis and necrosis in cardiomyocytes. J Biol Chem 2004;279:8290-8299.

16. Choi JY, Youn HJ, Kang JH, et al. Transthoracic echocardiographic assessment of adriamycin-induced cardiomyopathy in rats with a $15 \mathrm{MHz}$ transducer. J Korean Soc Echocardiogr 2000;8:78-86.

17. Choi YS, Park CS, Cho EJ, et al. The relation between acute adriamycin induced cardiomyopathy and apoptosis in rat: study using $15 \mathrm{MHz}$ high frequency transducer. J Korean Soc Echocardiogr 2002;10:35-43.

18. Uhm JS, Youn HJ, Park CS, et al. Comparison of adriamycin-induced cardiomyopathy in normotensive rats and spontaneously hypertensive rats. J Korean Soc Hypertens J 2006;12:23-30.

19. Rochette L, Guenancia C, Gudjoncik A, et al. Anthracyclines/trastuzumab: new aspects of cardiotoxicity and molecular mechanisms. Trends Pharmacol Sci 2015;36:326-348.

20. Salazar-Mendiguchia J, Gonzalez-Costello J, Roca J, Ariza-Sole A, Manito N, Cequier A. Anthracycline-mediated cardiomyopathy: basic molecular knowledge for the cardiologist. Arch Cardiol Mex 2014;84:218-223.

21. Minotti G, Menna P, Salvatorelli E, Cairo G, Gianni L. Anthracyclines: molecular advances and pharmacologic developments in antitumor activity and cardiotoxicity. Pharmacol Rev 2004;56:185-229.

22. L'Ecuyer T, Sanjeev S, Thomas R, et al. DNA damage is an early event in doxorubicin-induced cardiac myocyte death. Am J Physiol Heart Circ Physiol 2006;291:H1273-H128o.

23. Octavia Y, Tocchetti CG, Gabrielson KL, Janssens S, Crijns HJ, Moens AL. Doxorubicin-induced cardiomyopathy: from molecular mechanisms to therapeutic strategies. J Mol Cell Cardiol 2012;52:1213-1225.

24. Fu LX, Waagstein F, Hjalmarson A. A new insight into adriamycin-induced cardiotoxicity. Int J Cardiol 1990;29:15-20.

25. Minotti G, Ronchi R, Salvatorelli E, Menna P, Cairo G. Doxorubicin irreversibly inactivates iron regulatory proteins 1 and 2 in cardiomyocytes: evidence for distinct metabolic pathways and implications for iron-medi- ated cardiotoxicity of antitumor therapy. Cancer Res 2001;61:8422-8428.

26. Tokarska-Schlattner M, Zaugg M, Zuppinger C, Wallimann T, Schlattner U. New insights into doxorubicin-induced cardiotoxicity: the critical role of cellular energetics. J Mol Cell Cardiol 2006;41:389-405.

27. Schubert C, Hong S, Natarajan L, Mills PJ, Dimsdale JE. The association between fatigue and inflammatory marker levels in cancer patients: a quantitative review. Brain Behav Immun 2007;21:413-427.

28. Chiosi E, Spina A, Sorrentino A, et al. Change in TNF-alpha receptor expression is a relevant event in doxorubicin-induced $\mathrm{H}_{9 \mathrm{c} 2}$ cardiomyocyte cell death. J Interferon Cytokine Res 2007;27:589-597.

29. Youn HJ, Kim HS, Jeon MH, et al. Induction of caspase-independent apoptosis in Hyc2 cardiomyocytes by adriamycin treatment. Mol Cell Biochem 2005;270:13-19.

30. Zhang S, Liu X, Bawa-Khalfe T, et al. Identification of the molecular basis of doxorubicin-induced cardiotoxicity. Nat Med 2012;18:1639-1642.

31. Vejpongsa P, Yeh ET. Topoisomerase $2 \beta$ : a promising molecular target for primary prevention of anthracycline-induced cardiotoxicity. Clin Pharmacol Ther 2014;95:45-52.

32. Chung WB, Youn HJ, Choi YS, et al. The expression of cardiac ankyrin repeat protein in an animal model of adriamycin-induced cardiomyopathy. Korean Circ J 2008;38:455-461.

33. Levkau B, Schafers M, Wohlschlaeger J, et al. Survivin determines cardiac function by controlling total cardiomyocyte number. Circulation 2008;117:1583-1593.

34. Swain SM, Whaley FS, Ewer MS. Congestive heart failure in patients treated with doxorubicin: a retrospective analysis of three trials. Cancer 2003;97:2869-2879.

35. Menna P, Salvatorelli E, Minotti G. Cardiotoxicity of antitumor drugs. Chem Res Toxicol 2008;21:978-989.

36. Altena R, Perik PJ, van Veldhuisen DJ, de Vries EG, Gietema JA. Cardiovascular toxicity caused by cancer treatment: strategies for early detection. Lancet Oncol 2009;10:391-399.

37. Tan-Chiu E, Yothers G, Romond E, et al. Assessment of cardiac dysfunction in a randomized trial comparing doxorubicin and cyclophosphamide followed by paclitaxel, with or without trastuzumab as adjuvant therapy in node-positive, human epidermal growth factor receptor 2-overexpressing breast cancer: NSABP B-31. J Clin Oncol 2005;23:7811-7819. 
38. Perez EA, Suman VJ, Davidson NE, et al. Cardiac safety analysis of doxorubicin and cyclophosphamide followed by paclitaxel with or without trastuzumab in the North Central Cancer Treatment Group N9831 adjuvant breast cancer trial. J Clin Oncol 2008;26:1231-1238.

39. Armstrong GT, Oeffinger KC, Chen Y, et al. Modifiable risk factors and major cardiac events among adult survivors of childhood cancer. J Clin Oncol 2013;31:3673-3680.

40. Suter TM, Ewer MS. Cancer drugs and the heart: importance and management. Eur Heart J 2013;34:1102-1111.

41. Thavendiranathan P, Poulin F, Lim KD, Plana JC, Woo A, Marwick TH. Use of myocardial strain imaging by echocardiography for the early detection of cardiotoxicity in patients during and after cancer chemotherapy: a systematic review. J Am Coll Cardiol 2014;63(25 Pt A):2751-2768.

42. Tarr A, Stoebe S, Tuennemann J, et al. Early detection of cardiotoxicity by $2 \mathrm{D}$ and $3 \mathrm{D}$ deformation imaging in patients receiving chemotherapy. Echo Res Pract 2015;2:81-88.

43. Poulin F, Thavendiranathan P. Cardiotoxicity due to chemotherapy: role of cardiac imaging. Curr Cardiol Rep 2015;17:564.

44. Tamene AM, Masri C, Konety SH. Cardiovascular MR imaging in cardio-oncology. Magn Reson Imaging Clin N Am 2015;23:105-116.

45. Cardinale D, Sandri MT, Colombo A, et al. Prognostic value of troponin I in cardiac risk stratification of cancer patients undergoing high-dose chemotherapy. Circulation 2004;109:2749-2754.

46. Yu AF, Ky B. Roadmap for biomarkers of cancer therapy cardiotoxicity. Heart 2016;102:425-430.

47. de Azambuja E, Ameye L, Diaz M, et al. Cardiac assessment of early breast cancer patients 18 years after treatment with cyclophosphamide-, methotrexate-, fluorouracil- or epirubicin-based chemotherapy. Eur J Cancer 2015;51:2517-2524.

48. Park CS, Youn HJ, Cho EJ, et al. Cardioprotective effect of IGF-1 in mouse with adriamycin induced cardiomyopathy. Korean Circ J 2002;32:1116-1123.

49. Uhm JS, Chung WB, Yoon JS, Oh YS, Youn HJ. Effects of adriamycin and candesartan on the collagen and elastin of the aorta in rats. Clin Hypertens 2014;20:8.

50. Cardinale D, Bacchiani G, Beggiato M, Colombo A, Cipolla CM. Strategies to prevent and treat cardiovascular risk in cancer patients. Semin Oncol 2013;40:186-198. 